\title{
CHINA Y HONG KONG, 2017
}

\author{
Francisco Javier Haro Navejas \\ Universidad de Colima
}

\section{CRECIENTE MUNDIALIZACIÓN Y MULTIDIMENSIONALIDAD: MAYOR INFLUENCIA DE LOS ACTORES CHINOS}

El número de actores chinos en escenarios internacionales es cada vez mayor y su abanico de intereses también es creciente. Durante 2017 se fortalecieron dos de sus características esenciales: primero, la mundialización, están en prácticamente todo el planeta, segundo, sus campos de acción que, alentados por sus intereses, son multidimensionales. Durante el año pasado, trataron de posicionarse como una fuerza esencial para resolver problemas. Incluso, hacen todo lo necesario para involucrarse en escenarios de dominio tradicional de los poderes surgidos en la segunda posguerra. El mejor ejemplo de ello es la propuesta de Xi Jinping, presidente de China, compuesta de cuatro puntos ${ }^{1}$ para el conflicto entre Palestina e Israel: lograr la existencia de dos Estados basados en las fronteras de 1967 y el este de Jerusalén como capital palestina, finalizar el levantamiento de nuevos asentamientos judíos y terminar con la violencia contra los civiles, alentar la cooperación internacional para promover medidas pacíficas, promover la paz entre Israel y Palestina mediante el desarrollo y la cooperación. La propuesta, una de las primeras en materia de política exterior hechas por Xi a su llegada al poder en 2013, fue presentada el año pasado como algo bienvenido por las partes involucradas; incluso Israel aceptaría una mayor influencia de Beijing, por lo menos en la versión del enviado especial chino para la región, Gong Xiaosheng. ${ }^{2}$

\footnotetext{
${ }^{1}<$ www.timesofisrael.com/china-pushes-four-point-israeli-palestinian-peace-plan/>.

${ }^{2}<$ http://news.xinhuanet.com/english/2017-11/07/c_136732623.htm>.
} 
Algunos actores domésticos chinos se han mundializado cada vez más. Son escuchados e influyen en las decisiones que se toman en otros países. Éste ha sido un largo proceso, iniciado desde los años cincuenta del siglo pasado, pero ascendente de manera más rápida a partir del ingreso de China a la Asamblea General (AG) y al Consejo General (CG) de la Organización de las Naciones Unidas (ONU) a inicios de los setenta, además de catapultado por el incremento de las exportaciones a inicios de la década de 1990.

En un extremo, es posible observar el ascenso planetario de Ai Weiwei, miembro de la elite cultural, lo mismo por herencia que por derecho propio, ha tenido una relación muchas veces ambivalente con el Estado chino. Pese a todo, las desavenencias nunca han llegado a ser rupturas irreversibles y su interrelación con las fuerzas estatales está llena de matices. Artista plástico famoso en círculos relativamente pequeños de Estados Unidos y Europa, a veces más preocupados por la política que por el arte, se proyectó masivamente a partir de su participación en el diseño del famoso estadio Nido de Pájaros para los Juegos Olímpicos de Beijing, en 2008. A partir de la crisis de los refugiados sirios, Ai se ha dedicado a viajar por casi todo el planeta filmando, dando conferencias, recibiendo reconocimientos y exponiendo su obra, principalmente. Su principal instrumento de comunicación cotidiano es Instagram. Latinoamérica ha sido importante, donde sobresale Tijuana, México, pero también están otras ciudades como Buenos Aires, Argentina, y Santiago, Chile. Los resultados más distinguidos de su periplo, por lo menos por dos decenas de países, son: el documental Flujo humano, donde él actúa, y que se ha presentado en varios festivales, lo mismo de Europa que de América Latina, y que comercializa por Amazon; haberse convertido en actor político doméstico en algunos de los lugares que visita, como el caso de São Paulo, donde participó en protestas contra la censura; conectarse en Chile con los locales a partir de la relación, hace alrededor de seis décadas, entre su padre, Ai Qing, y Pablo Neruda. Incluso, de forma reciente, opinó sobre la situación en Cataluña.

No es el primer artista chino, plástico o de otra naturaleza, que se internacionaliza. Sin embargo, sobre todo durante 2017, Ai se diferenció del resto de sus pares, por rebasar su disciplina artística, su práctica militante y por carecer de una base china para realizar sus actividades. Ha transgredido las fronteras de otros artistas, tanto estéticas como políticas y espaciales. Él mismo dice que su vida es "[M]aravillosa. Puedo vivir en hoteles y viajar con 
mis colegas. Tengo a mi hijo conmigo. Tengo base en Berlín, pero viajo mucho". ${ }^{3}$ Por ejemplo, supera con mucho a Gong Li, actriz china globalizada pero discreta, y al taiwanés Ang Lee, artísticamente más universal que chino, lo que Ai no es, pero concentrado en su arte.

Entre los extremos están las personas de negocios. Se mueven entre el Estado y la sociedad, aunque tienden a estar más cerca de las instituciones estatales, las cuales les sirven para globalizarse. Ellos también cuentan con actores domésticos globalizados e influyentes. Ahora el sobresaliente es Ma Yun, conocido como Jack Ma, quien en 2017 visitó varios países donde se entrevistó o compartió el escenario con gobernantes como Vladimir Putin, Donald J. Trump, Justin Trudeau, Enrique Peña Nieto y Rodrigo Roa Duterte. Es un empresario-motivador-diplomático que lo mismo fortalece su empresa, Alibaba, que enseña cómo hacer negocios, llega a acuerdos con gobiernos y actúa en el cine al lado de Donnie Yen. Su mantra dominante durante el año anterior fue aseverar que ha llegado el momento de vender productos a los chinos, para lo cual promueve que exista internet de mayor velocidad y se compromete, como lo ha hecho con Trump y Peña Nieto, a apoyar a pymes de sus países para que exporten, con lo cual se crearían empleos. Para Alibaba el 11-11 ha dejado de ser el día chino de los solteros para convertirse en el Festival Global de las Compras y, a través de la plataforma Tmall, ha facilitado que empresas extranjeras lleguen a los consumidores chinos. El resultado son las ventas por poco más de 25 mil millones de dólares en un día.

Si bien el peso específico de las personalidades no es despreciable, el elemento sobresaliente es la internacionalización de un creciente número de empresas. El proceso se da de la mano de esfuerzos gubernamentales expresados en acuerdos bilaterales y multilaterales que abren el camino a las empresas. Existen casos específicos e importantes de internacionalización empresarial en Europa, principalmente. Sin embargo, Estados Unidos es el mercado más relevante en materia de inversión directa china. Lo es por lo menos por dos razones: la primera, de carácter político, es que sirve para construir percepciones positivas que dejen atrás la idea de una economía china que roba el trabajo a los

\footnotetext{
${ }^{3}<$ www.lanacion.com.ar/2080137-ai-weiwei-yo-era-la-persona-mas-controlada-de-china $>$.
} 
estadounidenses; la segunda, económica, está alentada por la racionalidad de estar cerca de los consumidores, con lo cual se reducirían los costos.

En 2017, en un campo dominado tradicionalmente por Estados Unidos, Japón y Alemania, los chinos se abrieron paso entre las principales compañías según Fortune. En números, dentro de las 500, China obtuvo el segundo lugar después de Estados Unidos, 132, con 115 empresas en la lista. Los lugares 2, 3 y 4 fueron ocupados por State Grid, Sinopec y National Petroleum, respectivamente. El primero fue Walmart, de Estados Unidos. Empresarios chinos han optado por la internacionalización, además de los casos e inversiones chinas en Europa, abordados abajo, el territorio estadounidense es el sitio preferido para invertir. Hasta octubre de 2017, había 93 empresas chinas activas financieramente en Estados Unidos, 41 en la National Association of Securities Dealers Automated Quotation (NSDAQ) y 52 en la New York Stock Exchange (NYSE). Los ramos dominantes son programas de computación y servicios asociados, comercio, medios, turismo, energía alternativa y telefonía celular, principalmente. En el denominado mercado de valores con transacciones electrónicas (over-the-counter market-OTC), existen 200 empresas de China entre las que se encuentran, sobre todo, compañías de la construcción y autopartes, bancos, servicios financieros, comerciales, bienes raíces, minería, transporte industrial, productores de alimentos. ${ }^{4} \mathrm{Un}$ punto de referencia para medir, al menos parcialmente, la participación china en estos mercados es contrastarla con la japonesa. Dentro de los dos primeros hay 14 empresas, sobre todo bancos, servicios financieros y compañías automotrices; mientras que en el segundo hay 321 participantes nipones, donde destaca el sector automotriz, las industrias químicas y electrónicas, los servicios financieros y los relacionados con la computación. Otros puntos de referencia son las empresas mexicanas, 14 y 27, respectivamente, mientras que las alemanas son 3 en la NYSE y 115 en el mercado OTC.

Entre los muchos ejemplos reales de inversión china están los de impulso a la pesca comercial o de desarrollo urbano planificado, como el que está en marcha en Cleveland, Texas, al norte de Houston. Ante la ausencia de fondos de cualquier nivel de gobierno, las autoridades del condado y los habitantes han buscado el financiamiento chino para mejor su entorno y darle mayor valor al mismo al construir un campo de golf, un centro comercial,

\footnotetext{
${ }^{4}<$ http://topforeignstocks.com/foreign-adrs-list/the-full-list-of-chinese-adrs/>.
} 
una alberca, entre otras instalaciones. ${ }^{5}$ Durante la visita a Beijing de Trump a inicios de noviembre de 2017, se dieron a conocer inversiones chinas por 250 mil millones de dólares. El sector atractivo para la firma de memorandos de intención fue el energético. El más llamativo para la prensa ha sido el de la empresa china Energy Investment, que invertiría 84 mil millones de dólares en Virginia Occidental en gas de lutita y manufactura química. Uno más es para desarrollar la industria de gas licuado en Alaska entre la empresa China Petroleum \& Chemical Corporation (Sinopec) y el gobierno de Alaska, donde se invertirían 43 mil millones de dólares. ${ }^{6}$ Ya desde el 19 de mayo del año anterior, el fondo soberano China Investment Corporation (CIC) inauguró una oficina en Nueva York para alentar una mayor inversión directa en Estados Unidos.

En el otro extremo de los actores domésticos mundializados que influyen sobre las decisiones de contrapartes de otros países está Xi Jinping, presidente de la República Popular China y de la Comisión Militar Central, secretario general del partido. El personaje real y simbólicamente más importante del sistema político chino ha sido designado a partir del XIX Congreso del Partido Comunista, en octubre de 2017, para formar parte del panteón teórico partidista con su "Pensamiento sobre el socialismo con características chinas para una nueva era", junto con las aportaciones de sus antecesores: "Los principios básicos del marxismoleninismo", "El pensamiento de Mao Zedong", "La teoría de Deng Xiaoping", "El importante pensamiento de la triple representatividad" (Jiang Zemin), y "El concepto científico de desarrollo" (Hu Jintao).

Trascendiendo la lectura de las hojas de té, que se centra en las dimensiones del poder de Xi, la trasferencia o no del mismo en 2022 y sus presuntas similitudes con Mao Zedong, lo relevante del personaje, dentro del contexto actual, se puede agrupar en el análisis de temas generales y comprobables. El primero es el creciente abandono de la autovictimización como táctica negociadora y creadora de identidad china, la cual es sustituida por un discurso en que el Estado chino se presenta como un poder global con agenda y medios para materializarla. Además, ofrece soluciones aparentemente factibles para solucionar problemas lo mismo globales que locales. Un segundo tema sería que, bajo la dirección de Xi, se han fortalecido

\footnotetext{
${ }^{5}<$ http://usa.chinadaily.com.cn/world/2017-08/17/content_30749022.htm>.

${ }^{6}<$ http://money.cnn.com/2017/11/09/investing/china-trump-business-deals/index.html $>$.
} 
los mecanismos institucionales para el ejercicio del poder, sobre todo el papel del partido y los diferentes componentes estatales, proceso discontinuo cuyos orígenes se remontan al IX Congreso partidario (1969); tercero, es posible afirmar que elementos simbólicos del ejercicio del poder, como inscribir la aportación, incluso el nombre del líder principal del momento en el programa y estatutos partidarios, es ya un elemento estructural y distintivo que fortalece al poder, le brinda legitimidad, lo institucionaliza y garantiza la continuidad en los términos negociados por los grupos políticos desde la época de Deng Xiaoping.

El proceso de construcción de legitimidad y fortalecimiento es complejo y antecede al congreso. Por ejemplo, a mediados de 2017, enfundados en camisas blancas de manga corta, algunos de los líderes políticos lanzaron una campaña a favor de la línea de masas, elemento esencial para el triunfo en 1949, componente esencial de la continuidad de las políticas del partido y muestra de la vinculación del liderazgo antes de 1949 con el actual.

En el discurso de Xi hay elementos de continuidad y ninguno de ruptura, lo cual fortalece la institucionalidad estatal. Los ejes discursivos de Xi son el combate a la corrupción, la modernización del ejército, la renuncia a la hegemonía global, la defensa de la soberanía territorial y los buenos resultados obtenidos con el concepto "un país, dos sistemas". Hay componentes particulares en su discurso, pero que no rompen con la tradición. Ha establecido fechas para la modernización socialista y para el momento en que China será un país socialista grande, además de explicar que el rejuvenecimiento del país solamente será posible bajo el liderazgo del partido, entre otros planteamientos. En el discurro de Xi también se habla de respetar las leyes de la naturaleza para evitar que ésta se vuelva en contra de los seres humanos, con lo cual se sitúa en un discurso dispuesto a resolver uno de los grandes problemas de China, el de la contaminación, lo mismo en términos reales que de percepción. Sin embargo, lo trascendente es asumir el papel de un poder global.

\section{Et ego te absolvo...}

En 2017 China llegó a ser explícitamente una potencia y parte de un binomio de poderes globales. Para ser un poder en el sistema internacional se puede carecer de algunos atributos necesarios. Viceversa, se puede contar con los atributos, pero se es poder en tanto que se tiene el reconocimiento de los demás de ser tal. El Estado Vaticano, con el amor papal 
profesado a China, ${ }^{7}$ fortalece al gobierno a nivel global, pero de manera insuficiente. Si a fines de los años sesenta e inicios de los setenta el Estado chino podría haber carecido de algunos atributos para ser una potencia global, llegó a serlo por el reconocimiento de la Casa Blanca debido a necesidades geopolíticas de la época. Con el discurso de su visita a Beijing en noviembre del año pasado, Trump colocó a China como poder global. Es ahora su interlocutor por excelencia, la relación bilateral más importante a la cual todos observan.

Por ahora no es posible saber si fue un diálogo de sordos desconocedores del lenguaje de señas o con dominio de diferentes y mutuamente incomprensibles lenguajes. En los discursos aparece que, para $\mathrm{Xi}$, el Pacífico es suficiente para ambos, ${ }^{8}$ no hay necesidad de pelear. Días después, en la reunión anual de líderes del Foro de Cooperación Económica Asia Pacífico (APEC), Xi presentó un discurso de un líder con propuestas, ${ }^{9}$ pero sin el espíritu combativo respecto a Estados Unidos mostrado en Perú en 2016. Mientras, para Trump la agenda interna es la importante, por ello responsabilizó del déficit comercial con China únicamente a sus antecesores, ${ }^{10}$ lo cual parece incluir a republicanos y demócratas. El presidente estadounidense absolvió, literalmente, a sus anfitriones, quienes lo único que habrían hecho fue aprovechar la puerta que les abrieron en beneficio de su población, por lo cual merecen todo el crédito posible.

Ello no quiere decir que no haya algo de política internacional, así sea su retórica contra Corea del Norte.

\section{CHINA, UNA POTENCIA EN LA ALFOMBRA ROJA: PERCEPCIONES, DISCURSO Y ACCIONES MULTIDIMENSIONALES}

En Beijing saben que la percepción es (casi) todo. Los líderes se han preocupado por agradar a los extranjeros. Han organizado y otorgado prácticamente todo lo imaginable: competencias deportivas, carreras de autos, reuniones de líderes gubernamentales, congresos,

\footnotetext{
${ }^{7}<$ http://www.dailymail.co.uk/wires/afp/article-4761204/Vatican-official-says-Pope-loves-China.html $>$.

$8<$ www.whitehouse.gov/the-press-office/2017/11/09/remarks-president-trump-and-president-xi-chinajoint-press-statement $>$.

${ }^{9}<$ http://news.xinhuanet.com/english/2017-11/11/c_136745175.htm>.

${ }^{10}<$ www.whitehouse.gov/the-press-office/2017/11/09/remarks-president-trump-business-event-president-xichina-beijing-china $>$.
} 
exposiciones, cursos, préstamos, donaciones, entre otra muchas acciones y hechos. El trato dado a Trump en Beijing, en noviembre de 2017, es una muestra de ello. Fue excelso, incluso en ceder en un menú sin complicaciones para un gusto simple.

Los resultados de sus políticas han rendido frutos. En el índice marca país (Country Brand Index), ${ }^{11}$ donde Japón ocupa el lugar número 1, Argentina el 42, Brasil el 43, y México el 55, China ha escalado hasta el lugar 28. Además, su capital es considerada como la tercera ciudad más influyente en el mundo.

En China van a seguir explorando y explotando el tema de las percepciones, donde quiera que sea posible y necesario. Para ello han relanzado dos veces una plataforma existente desde medios masivos de comunicación, China Global Television Network. Esta empresa, existente desde 2002, sería la voz y la mirada del Estado chino sobre lo que acontece tanto dentro como fuera de sus fronteras compitiendo con la pionera Xinhua. La iniciativa fue relanzada por primera vez el 31 de diciembre de 2016, con la presentación de una aplicación; posteriormente la presentaron con lo más nuevo de la tecnología para que sus audiencias "vieran la diferencia".

También van a tratar de caminar por la alfombra roja.

La presencia de una película china en los premios Oscar no es nueva. Sin embargo, la selección oficial para 2017, en la categoría de mejor película en lengua extranjera, podría parecer por lo menos inadecuada. Lobo guerrero 2 sobresale entre las 92 presentadas, sobre todo las de Taiwán, Small Talk, y Hong Kong, Mad World. Tradicionalmente, en esta categoría sobresalen los filmes que cinematizan una problemática social enriquecida artísticamente.

Lobo... es la narrativa cinematográfica de una autopercepción y de por lo menos dos aspiraciones. El recurso narrativo utilizado es uno de las más viejos del cine de acción estadounidense: el héroe actúa para alcanzar un bien mayor fuera de las instituciones. Los productores, "los chinos", se (auto)perciben como una potencia planetaria cuyos ciudadanos enfrentan, debido a las buenas obras que realizan, ataques injustificados de actores ilegales no-gubernamentales. Las aspiraciones son por lo menos dos, sin contar la financiera, que se

\footnotetext{
${ }^{11}<$ www.futurebrand.com/thought-leadership $>$.
} 
da por sentada. Primera, hacer que su percepción sea compartida globalmente, partir de la conquista de simpatías vía la pantalla; y segunda, crear una narrativa, a diferencia de la estadounidense, sin el elemento histórico, la cual construye un espacio de acción e intereses chinos en partes específicas del mundo.

Con la ayuda del cine, surgiría así un elemento del afianzamiento chino como poder global. Gracias a la pantalla, se añade un elemento a la compleja relación con Estados Unidos, pero, sobre todo, el gobierno de Beijing levantó un trampolín para ser aceptada China socialmente como una potencia global.

Durante 2017, vimos un liderazgo chino, encabezado por Xi Jinping, completamente decidido a confirmar e incrementar su presencia como poder global, tanto dentro como fuera del sistema internacional. Estuvo presente con propuestas en prácticamente todos los foros $\mathrm{y}$, como desde los años ochenta, fortaleció sus múltiples relaciones bilaterales. La única excepción importante fue la ausencia de Xi Jinping en la Asamblea General (AG) de la Organización de Naciones Unidas (ONU) de septiembre en Nueva York. Sin embargo, el 18 de enero de 2017, además de ser un personaje importante en el Foro Económico Mundial, ofreció un discurso ante ese organismo en Ginebra. Entre otros tópicos, defendió el multilateralismo y sus políticas de cooperación, mediante las cuales han otorgado ayuda por 400 mil millones de renminbis.

Así como en septiembre de 2016 China fue sede de la reunión del G-20, un año después lo fue de los BRICS. El fortalecimiento como poder no sucede sin contratiempos, como los que representan la tensa situación en la península coreana y los problemas internos, que buscaron delinear soluciones en el XIX Congreso del Partido Comunista, en octubre de 2017.

Uno de los logros más importantes del gobierno chino del año anterior se relaciona con la pobreza. Sobre la base doble de percepciones positivas y hechos medibles, el Banco Mundial (BM) reconoció que China ha logrado que más de 800 millones de personas hayan salido de la pobreza gracias al rápido crecimiento de la economía. La institución constata que la china es la segunda economía más grande del mundo y la que más ha contribuido al crecimiento económico global desde la crisis financiera de 2008. El BM asegura que, pese a ello, se trata de una economía en desarrollo, debido a su bajo ingreso per cápita. Junto con luchar por ser reconocida como una economía de mercado, el que no se les clasifique como 
economía desarrollada es una de las grandes aspiraciones gubernamentales en Beijing. Es inevitable no ver que los logros son producto de resultados positivos considerablemente tangibles, pero también que son producto del trabajo de los negociadores chinos dentro de la institución.

\section{CHINA ANTE UN INEXISTENTE VACÍO DE PODER: UN CINTURÓN Y UN CAMINO}

El gobierno de Xi percibió el triunfo electoral de Donald J. Trump, sustentado en propuestas comerciales proteccionistas y en un abandono casi total del institucionalismo multilateral, como la creación de un vacío de poder global que Beijing podría llenar con facilidad, con muchas iniciativas específicas, más o menos regionales, y grandes cantidades de dinero. La percepción de Beijing se enfrentó a realidades que no se ajustaban cabalmente a la misma. Los nuevos enfoques de táctica estadounidenses no han sido abandono, ni en lo bilateral ni en lo multilateral; además, los gobiernos, salvo en aspectos y casos particulares, no cambiaron sus preferencias de forma automática. No se han plegado masivamente a Beijing.

Xi se ha encargado de explicitar que son un poder mundial, lo que lo diferencia de todos sus antecesores, que se esforzaron en negar que eso fuera posible, por lo menos en su presente. La conjunción de elementos medibles, objetivos, y de autopercepción, subjetivos, hace posible ver que la República Popular China es un poder global, pero han sido insuficientes para que el mundo se sume a sus políticas. Los cortejos son mutuos y los gobiernos buscan incrementar sus ganancias sin comprometerse totalmente con alguna de las potencias.

A diferencia de Estados Unidos, China no administra un orden global ni crea gran parte de la agenda del mismo. Trata de construir su propia versión a partir de iniciativas conectadas entre sí, no siempre sólidamente. A partir de septiembre de 2013, en un discurso ante universitarios de Kazajistán, Xi propuso la iniciativa "Un cinturón y un camino" (yidai yilu 一带一路), que quedó plasmada en el documentos “Vision and Actions on Jointly Building Silk Road Economic Belt and 21st-Century Maritime Silk Road", del 28 de marzo de 2015. 
La iniciativa no prosperó sustancialmente. Después, en 2017, el gobierno de Xi la retomó con gran ímpetu. La idea es unir el Este de Asia desde uno de sus espacios más meridionales, Filipinas, y Hong Kong, hasta Europa y África, lo cual implica cubrir todo lo que está entre esas antípodas siguiendo diferentes rutas de comunicación: una iría desde el extremo sur del Este hacia el Mediterráneo tocando a su paso el Sudeste Asiático, tanto insular como continental, a través del estrecho de Singapur, el Océano Índico y el Golfo Pérsico, con lo cual se conectaría también con el continente indio, el este y norte africanos y el oeste asiático. Una variante de esta ruta, con los mismos destinos y sitios a cubrir, partiría de Hong Kong y otra iría al oeste de África y al este sudamericano. Una segunda ruta iría desde las costas norteñas continentales chinas hasta la parte norte del continente indio a través del Sudeste, sobre todo por Myanmar y Bangladesh. Otra, que tendría una bifurcación, saldría igualmente del norte chino a través de Mongolia y Rusia hacia Europa, sobre todo el norte; mientras que, a partir de Asia Central, otra bifurcación iría hacia el Mediterráneo. Las rutas cubrirían, por mar y tierra, alrededor de seis decenas de países, con la pretensión de alentar el comercio y la inversión, mejorar la infraestructura y la integración financiera, y favorecer los intercambios culturales.

Beijing pretende insertar el proyecto en el marco de la Carta de la Organización de las Naciones Unidas (ONU), lo plantea abierto a cualquier institución y país, además de que, si bien es su propuesta, se asume como portavoz de otros al aseverar que se trata de una "aspiración de todos los países a lo largo de la ruta". ${ }^{12}$

Es un tipo ideal multidimensional en actores y objetivos, apuntalado por el desembolso ya realizado de 900 mil millones de dólares. Cada año China dedicaría 150 mil millones de dólares a la construcción de infraestructura en diferentes partes del globo: puertos, puentes, carreteras, entre otros. A lo que habría que agregar 8.7 millones en asistencia para las economías más pobres.

El aliento por el reacomodo de fuerzas global y el nuevo enfoque puesto en práctica por la Casa Blanca llevó a Xi a organizar y presidir una reunión de dos días en Beijing, en mayo de 2017. Asistieron 29 jefes de Estado, entre los cuales se encontraban Vladimir Putin, de Rusia; Nawaz Sharif, primer ministro de Pakistán; Hailemariam Desalegn, primer

\footnotetext{
${ }^{12}<$ http://en.ndrc.gov.cn/newsrelease/201503/t20150330_669367.html $>$.
} 
ministro de Etiopía; Philip Hammond, entonces secretario de Estado para Asuntos Exteriores del Reino Unido; Najib Razak, primer ministro de Malasia; e incluso estuvieron presentes Michelle Bachelet, mandataria chilena, y Mauricio Macri, de Argentina. ${ }^{13}$ Para estos dos presidentes el encuentro fue muy fructífero en lo bilateral.

Una presencia importante, desde el punto de vista geopolítico, fue la de Recep Tayyip Erdogan, presidente de Turquía, percibida como gozne entre Europa y Asia, y por lo mismo clave para el proyecto chino.

La lista con los desniveles jerárquicos de los presentes, muestra las diferentes intensidades, interés recibido por la propuesta, que van del acercamiento de Londres que busca opciones para su futuro incierto por la salida de la Unión Europea (UE) a la aceptación total de Islamabad, pasando por la búsqueda de Moscú de quedar fuera del orden internacional propuesto, sobre todo porque toca sus espacios históricos en Asia Central, pero que llegaría hasta la Rusia estrictamente europea y sus zonas de influencia ahora débil en ese continente, en la antes llamada parte oriental. Los representantes del Sudeste Asiático fueron muy importantes, como el caso malayo, también estuvo el presidente filipino Rodrigo Duterte y se contó con la presencia de Lee Hsien Loong, primer ministro de Singapur.

Durante este año, 2017, Beijing ha mostrado tener los recursos financieros y las ideas para proponerse encabezar ideas globales y crear un entorno internacional alternativo al existente. Sin embargo, enfrenta cuatro fuerzas que contrarrestan sus esfuerzos políticos: la falta de apoyo de la UE, la oposición franca de India a la iniciativa china, además del contexto geopolítico incierto y las dificultades objetivas para materializarlas. Las dos primeras son las más importantes.

Sin existir lo que pudiera ser una posición oficial, por lo menos desde 2015, la UE ha prestado atención al proyecto chino, el cual ha demandado que se negocie con la participación en el mismo de todos los posibles involucrados y que se realice con transparencia, actitud que se repitió en mayo de 2017. Si bien hay sectores, sobre todo ligados al comercio, interesados en acercarse a la iniciativa china, los gobiernos, tanto en lo individual como en lo colectivo, siguen sin posición explícita y favorable a Beijing. Las preocupaciones europeas

$13<$ https://www.theguardian.com/world/2017/may/14/china-xi-silk-road-vision-belt-and-road-claimsempire-building $>$. 
ahora son las mismas de 2015, sobre todo la relacionada con la transparencia. Las ausencias dicen más que las presencias, sobre todo la de los jefes de Estado de Francia y Alemania. Además, existen fuertes críticas europeas a la propuesta de Beijing. Por ejemplo, quien fuera presidente de la Cámara de Comercio de la UE en China, Jörg Wuttke, reafirmó que el proyecto gubernamental ha "sido secuestrado por las compañías chinas, las cuales lo usan para evadir los controles al capital, contrabandear dinero disfrazado como inversión y sociedades internacionales". ${ }^{14}$

Diferentes actores indios, empezando por el gobierno, tienen dificultades serias para aceptar una iniciativa multidimensional como la de "un cinturón y un camino", la cual es asfixiante para Delhi. Los chinos se instalan en los espacios históricos de los indios, el Océano Índico y sus fronteras; fortalecen sus lazos con su rival más fuerte, Pakistán, y con su siempre posible aliado, Daca, o más al sur con Colombo, y al norte con Nepal. Los chinos podrían acabar con el statu quo: el papel indio como potencia regional está cuestionado; finalmente, pero no es lo único, se apoderan del comercio y se convierten en el motor del crecimiento económico regional desplazando a todas las iniciativas indias en materia económica y de conectividad. Los cuestionamientos de Delhi giran alrededor de su percepción de que los chinos violan su soberanía, lo cual tiene que ver con los 50 mil millones de dólares destinados por China al corredor económico con Pakistán, y porque han solicitado abrir el dialogo con China, pero no han recibido respuesta alguna. La ausencia india en la reunión de mayo de 2017 muestra que, para Delhi, no se trata de construir puentes de cooperación, más bien es acerca de la distribución del poder.

En China, al menos en alguna versión de prensa, se consideró que la actitud india era una táctica para enfrentar problemas políticos internos. ${ }^{15}$ Las dificultades entre Beijing y Delhi fueron considerables, sobre todo en junio y agosto de 2017, por los enfrentamientos entre soldados de ambos ejércitos. India protege un territorio que considera es de Bután, pero que los chinos reclaman para sí. La disputa fue alrededor de Donglang, en el valle de Chumbi en los Himalaya, entre Bután y China.

\footnotetext{
$14<$ http://edition.cnn.com/2017/05/13/asia/china-belt-and-road-forum-xi-putin-erdogan/index.html>.

$15<$ http://timesofindia.indiatimes.com/india/indias-objections-to-obor-a-show-for-domestic-politics/article show/58697301.cms>.
} 
Una de las relaciones más complejas sostenidas por Beijing es la que mantiene con Delhi. Roces, desconfianza, intentos chinos de intervenir internamente al apoyar en diferentes momentos a algunos grupos, disputas comerciales, enfrentamientos fronterizos y competencia por ser la potencia regional son algunos de los componentes de la relación bilateral. En el lado opuesto está el marco de negociación y acuerdo que ofrecen los BRICS y que amortigua las diferencias. A fines de agosto, justo días antes de la reunión de los cinco países, ambos gobiernos llegaron a un acuerdo para aliviar las tensiones en el punto más conflictivo desde 1962, sobre el cual han negociado y renegociado varias veces.

La X Cumbre, bajo la dirección de Beijing, se efectuó en Xiamen, Fujian, China, y culminó el 8 de septiembre. Su declaración final ${ }^{16}$ cubre múltiples aspectos, los cuales rebasan lo meramente económico y abordan temas de seguridad. Los temas centrales fueron los de la cooperación, el papel activo jugado por los BRICS a favor del desarrollo económico y la necesidad de combatir al terrorismo de forma multilateral bajo la dirección de la ONU, cosa que aceptan en otras áreas. Uno de los principales objetivos planteados fue el de la puesta en marcha del Diálogo de Mercados Emergentes y Economías en Desarrollo para la Materialización de la Agenda para el Desarrollo Sustentable 2030. Así como el marco de este grupo permitió limar asperezas entre chinos e indios, el encuentro también sirvió parta invitar a potenciales aliados, como podrían ser México y Tailandia.

El gobierno chino mueve muchas piezas, dinero e ideas, para asentarse como poder global. Una de ellas fue el establecimiento, el 1 de agosto de 2017, de una base militar en las costas de Yibuti, sitio estratégico ya utilizado por Arabia Saudita, Estados Unidos, Francia y Japón. Para China es vital en su esquema general de la expansión de su poder y de acceso dentro del continente africano, así como al Canal de Suez. Este movimiento, relativamente limitado, esencialmente militar, es consistente con el proyecto de la también llamada nueva ruta de la seda.

\footnotetext{
${ }^{16}<$ https://www.brics2017.org/English/Headlines/201709/t20170908_2020.html $>$.
} 


\section{AMÉRICA DEL NORTE}

\section{Beijing-Washington, más allá del discurso beligerante de Trump}

En 2017, las relaciones entre Estados Unidos y China resultaron más tersas de que lo podría haberse esperado a partir del encuentro entre Xi y Trump en Florida, en abril de ese año. A ello ha contribuido el turismo creciente. Por ejemplo, Los Ángeles ya recibe un millón de turistas chinos anuales. También ha sido clave el trabajo de décadas de ambas burocracias, las cuales han cumplido con sus funciones, sobre todo para culminar con éxito negociaciones iniciadas en la administración de Barack Obama. Los frutos, como se mostró antes, se observan en la creciente inversión y en todos los proyectos existentes.

La contribución a la tersura también debe atribuirse a los funcionarios chinos, principalmente a $\mathrm{Xi}$, que cuentan con políticas multidimensionales y diferenciadas que les permiten diversificar sus intereses, lo cual posibilita el aumento de sus ganancias sin necesidad de recurrir de forma excesiva a la violencia, aunque sea verbal.

Con Trump, los chinos han jugado el juego de la cooperación, no el de la gallina. Los resultados han sido benéficos para ambas partes. La visita exitosa de Trump en noviembre estuvo precedida por acontecimientos importantes y resultados positivos a lo largo del año.

La expectativa estadounidense es la de una mayor apertura en servicios bancarios y financieros a ambos lados del Pacífico. ${ }^{17}$ En mayo firmaron un acuerdo bilateral mediante el cual se levanta la prohibición de que los estadounidenses exporten carne de res; a su vez, los chinos podrán exportar pollo cocido, se aprobará la comercialización de ocho productos biológicos, China podrá importar gas natural licuado, las firmas financieras ofrecerán calificaciones crediticias, no se sancionará en Estados Unidos al banco Shanghai Clearing House, el monopolio de UnionPay_en las tarjetas se acaba y entrarán lo mismo Visa que Mastercard, no se discriminará a los bancos chinos y China emitiría, antes del 16 de julio de 2017, bonos del Estado y licencias de negociación. ${ }^{18}$

\footnotetext{
$17<$ http://edition.cnn.com/2017/05/11/politics/china-us-beef-imports-trade-deal/index.html $>$.

$18<$ http://www.bbc.com/mundo/noticias-internacional-39897388>.
} 
Incluso el gobierno de $\mathrm{Xi}$ ha llevado a su contraparte estadounidense a terrenos incómodos, que le son ajenos y sobre los cuales no quiere actuar, pero sobre los que las burocracias de ambos países han trabajado adecuadamente. Uno de ellos es el de las relaciones pueblo-pueblo, parte esencial del enfoque multidimensional de los chinos. En este campo pretenden contar con apoyo social para que las relaciones bilaterales se fortalezcan, así como alcanzar acuerdos en ciencia y tecnología, salud, cultura deportes, educación, administración local y protección ambiental. Ambos gobiernos llegaron a muchos acuerdos, como el llamado Doble 100 000, similar a lo que en México se conoció como Proyecta 100 000. Mediante éste, cada uno intercambiará ese número de estudiantes durante cuatro años. Además, acordaron cooperar para realizar investigación sobre sida y ayudar a países africanos a crear un sistema de salud para combatir el ébola.

En Estados Unidos hay, sobre todo en medios académicos, críticas a los chinos y a las políticas estadounidenses respecto a ellos. Dos son los principales motivos de insatisfacción. El primero es que, al dar prioridad a los ingresos económicos y aceptar indiscriminadamente estudiantes provenientes de China, sobre todo por capacidad financiera, el nivel académico tendería a bajar. Los recién llegados no tienen gran interés en estudiar, plagian e inclusive pagan a otros para que hagan sus obligaciones académicas. El segundo, con mucho, rebasa el ámbito académico. Se trata del avance de los llamados Institutos Confucio, diseñados para la enseñanza del putonghua (lengua común o chino oficial) y la transmisión de la cultura china. Son financiados por el gobierno chino y su éxito se basa, de entrada, en que las universidades locales carecen de fondos gubernamentales y por ello aceptan los provenientes de Beijing. Los críticos sostienen que sus actividades son políticas y que minan libertades estadounidenses esenciales, como la de expresión.

Para Beijing, lo mejor fue lograr que en ambas negociaciones, la económica y la cultural, Washington aceptara decir que tomará en cuenta la iniciativa "un cinturón y un camino" para, en el vocabulario chino, levantar una comunidad con un destino compartido y preocupada por los temas de salud.

Momentáneamente, el mayor punto de conflicto bilateral ha sido en relación con Corea del Norte. La percepción de Trump es que Pyongyang sobrevive gracias a Beijing. Una vez que éste deje de prestarle apoyo, se derrumbará. 
Beijing ha adoptado una posición flexible ante la situación en la península, más por las presiones de la Casa Blanca que por las resoluciones de la ONU. El primero ve una situación más compleja que no se resuelve simplemente con bombas. Ante todo, se preocupa por la estabilidad del Este de Asia, en especial por la de Dongbei (noreste chino), que quedaría vulnerable ante una explosión en el norte peninsular, sobre todo ante un previsible pero poco controlable flujo de refugiados. A finales de septiembre de 2017, el gobierno chino ordenó a las empresas coreanas, asociadas o no con sus nacionales, a cerrar a más tardar en enero de 2018. El 5 agosto, el Consejo de Seguridad de la ONU, con 15 votos a favor, adoptó medidas económicas contra Corea del Norte dirigidas a evitar que reciba ingresos por exportaciones, empresas o bancos. Poco más de un mes después, el 12 de septiembre, bajo las propuestas de Estados Unidos, el organismo profundizó las sanciones, entre las que destacan impedir la exportación de petróleo al norte y la existencia de empresas conjuntas, transferencia de tecnología, inversiones y el bloqueo al envío de remesas. Por esto, a China no le quedó margen de maniobra y se plegó, al menos parcialmente, sin satisfacer completamente a Trump. Además, existe la amenaza de que en algún momento Washington pueda sancionar a empresas y bancos chinos que tienen negocios en Pyongyang.

A finales de septiembre de 2017, Rex Tillerson, secretario de Estado, viajó a Beijing para preparar la agenda del viaje de Trump en noviembre, en ella sobresalen el tema del déficit comercial estadounidense y el ánimo de ejercer presión política sobre el gobierno chino. La expectativa es que éste ayude a ahogar económicamente aún más a Pyongyang, de la que China es su principal cliente. Los puntos finos económicos estuvieron a cargo de Wilbur Ross, secretario de Comercio, quien visitó la capital china el 25 del mismo mes, días antes que Tillerson. Ross expuso temas relacionados con proteccionismo-acceso a mercados, propiedad intelectual, tarifas, pero sobre todo con el consabido déficit comercial y el trato recíproco a las empresas de su país. Hasta ahora la oficina de Ross se ha contentado con impulsar investigaciones relacionadas con violaciones a los derechos de propiedad intelectual y relacionadas con pretendidos subsidios al acero.

\section{Canadá, la puerta americana de la ruta de la seda}

La relación bilateral sino-canadiense es estable y poco conflictiva. En el verano de 2017 ambas naciones dieron a conocer un estudio donde se concluye que las dos son economías 
complementarias. Lo más importante es que firmaron un acuerdo para proteger y alentar la inversión mutua. Los chinos han profundizado la relación gracias al primer ministro Justin Trudeau, quien solicitó la aceptación de su país en el Banco Asiático de Inversión en Infraestructura.

Uno de los trabajos diplomáticos que hacen con mayor eficiencia los chinos en Canadá es a nivel provincial. Han logrado acuerdos con Nueva Escocia para convertirla en la llamada Puerta del Atlántico, y con Columbia Británica, la Puerta del Pacifico, y que sean parte de la iniciativa "un cinturón...". Sobresale el hecho de que el parlamento provincial de Toronto, el 26 de octubre de 2017, seguido por el de Manitoba, aprobó una moción según la cual cada 13 de diciembre se conmemorará la llamada Masacre de Nanjing. La iniciativa fue presentada por la legisladora Soo Wong, nacida en 1962 en Hong Kong y emigrada a Canadá a los 8 años de edad. Preocupada por temas de educación y de salud, e inclusive por aspectos relacionados con prisiones, su relación con temas chinos tan específicos parece inexistente más allá de su argumentación de que el tema debe ser enseñado a los estudiantes canadienses.

Si bien a nivel gubernamental las relaciones funcionan adecuadamente, a nivel social esto no ocurre y es algo que preocupa a la diplomacia china. De acuerdo con su embajador, Lu Shaye, el gobierno no debería hacer caso a los periodistas que escriben sobre los derechos humanos en China y tendría que concentrarse en negociar un tratado de libre comercio con Beijing. Trudeau coincide en que el tratado debería ser una prioridad y los diplomáticos ya han avanzado en las conversaciones con China.

\section{México, inesperado objeto del deseo}

En 2017, por un tiempo breve, México fue visto como un objeto de deseo por Beijing. Según su visión de que existía un vacío de poder, el vecino sureño ya no sería necesario para Washington. El estilo agresivo de negociación de Trump no significa que vaya a dejar ir a su adversario, o que éste lo vaya a abandonar. Detrás de él vienen los que negociarán de manera más suave y con quienes será posible llegar acuerdos, en este caso la renegociación del Tratado de Libre Comercio de América Latina. Del otro lado de la moneda, también se habría calculado erróneamente la intención gubernamental mexicana de alejarse de Washington, lo cual no ha sido planteado ni siquiera hipotéticamente por las elites mexicanas gobernantes y no pocas económicas en tiempos de Trump. 
A finales de junio de 2017, el gobierno chino sobre todo mediante su embajada en la capital mexicana, fue insistente respecto a su disposición, sin preocuparse por terceras opiniones, a negociar con México un tratado comercial. Pensaron, es de suponer, que Los Pinos abrazarían la idea china en virtud de los desplantes de la Casa Blanca. Desde por lo menos diciembre de 2016, el embajador Qiu Xiaoqi mostró su disposición a fortalecer la relación con los mexicanos ante la posibilidad de quedarse sin TLCAN y debido a la desaparición del TPP.

Tanto desde la Ciudad de México como desde Beijing le han hecho muchos guiños a Enrique Peña Nieto, presidente mexicano, quien estuvo en la reunión de Xiamen de los BRICS. Los temas que más ocuparon a Peña fueron el TLCAN, en lo general, así como la llegada de instituciones financieras chinas y un nuevo vuelo desde Guangzhou en 2016, en particular.

Ante el sismo del 19 de septiembre de 2017 el gobierno chino mostró su solidaridad con los mexicanos y donó alrededor de ocho millones de dólares, tanto en efectivo como en diferentes materiales, como casas de campaña. La Cruz Roja de ese país hizo otro tanto, donó 100 mil dólares y la propia embajada dio 20 mil dólares al Sistema Nacional para el Desarrollo Integral de la Familia, organismo encabezado por la esposa del presidente, Angélica Rivera.

México, como el resto del mundo, aún no ha visto un avance global e impresionante de las marcas chinas, similar a lo que pasó con las japonesas a fines de los años setenta e inicios de los ochenta, o con las coreanas durante los primeros años de la década de 1990. No obstante, en el ramo automotriz, FAW y BAIC en los últimos meses han tratado de fortalecer su presencia, sobre todo la segunda, que arribó apenas en 2016 y que ya cuenta con "embajadas" en 16 estados mexicanos. En la telefonía los avances de Huawei y Xiaomi son considerables, por ejemplo, la segunda pretende posicionarse con un modelo que competiría con Samsung y Apple con precios considerablemente más bajos.

Las relaciones con México son complicadas por muchas razones, por ejemplo, las económicas que implican una carga sentimental. Los ejemplos más notorios son los relacionados con la intención china, como de otros países, de patentar el nopal o el hecho de que los principales productores, $75 \%$ mundial, de cempasúchil son los chinos. 


\section{LA AGENDA CHINA CON LA UNIÓN EUROPEA}

Las relaciones con Europa se han realizado a dos niveles por lo menos. Uno es el de la agenda que la burocracia de la UE trata de construir con Beijing, y la otra es la gubernamental bilateral, la cual es diferenciada por el lugar que ocupa Alemania —el pilar regional—, en la misma, y el que empieza a ocupar el Reino Unido, precisamente, por lo contrario.

El 13 de septiembre, Jean-Claude Juncker, el presidente de la Unión Europea, brindó su informe del año 2017. En él no hay ninguna referencia explícita a China. En el discurso están, en la parte comercial y por orden de aparición, Japón, México, "países sudamericanos", Australia y Nueva Zelanda. Con el primero tienen un acuerdo político para alcanzar una sociedad económica, con los latinoamericanos esperaban concluir negociaciones a fines de 2017, y con los dos últimos quieren iniciar negociaciones comerciales.

Sin embargo, se dejó claro que no son librecomerciantes "ingenuos", un concepto cuya acuñación se adjudica a Emmanuel Macron. Asegura que, por ello, proponen un nuevo marco para el monitoreo de inversiones extranjeras. El planteamiento está dirigido implícitamente a los chinos y asegura que "[S]i una empresa extranjera, propiedad del Estado desea comprar un puerto europeo, parte de nuestra infraestructura de energía o una firma de tecnología de defensa, esto solamente podrá pasar con transparencia, con escrutinio y debate". ${ }^{19}$ El objetivo es proteger la seguridad colectiva. Además del tema de la seguridad, está el hecho de que, de acuerdo con los europeos, sus contrapartes chinas no ofrecen reciprocidad y son proteccionistas en materia de inversión, lo cual se aprecia tanto en la banca como en la industria automotriz.

Comparado con lo anterior, sobre todo con la imposibilidad de que en 2017 se firmara un acuerdo en materia de inversión, e impedir más transferencia de tecnología a China, el menor de los problemas que los europeos tienen con los chinos es el de la importación de productos baratos y de mala calidad, pero ante lo cual parecen no poder ponerse de acuerdo; por lo menos no fue así ni con el informe de Juncker.

\footnotetext{
${ }^{19}<$ http://europa.eu/rapid/press-release_SPEECH-17-3165_en.htm>.
} 
Para enfrentar a China, en los meses recientes algunas empresas europeas se han unido de diferentes maneras, tal y como lo hiciera la finlandesa Nokia al comprar Alcatel-Lucent en una negociación iniciada en 2015 para competir con marcas como ZTE y Huawei. La operación aún es una promesa de éxito.

En 2017 se produjeron por lo menos dos operaciones importantes en la industria europea. El 19 de septiembre se hizo público que la alemana Thyssenkrupp AG y la india Tata Steel Ltd. firmaron un memorándum de entendimiento, transacción en espera de la sanción de la UE, para formar el conglomerado Thyssenkrupp Tata. Competirían con una europea, ArcelorMittal, y con los chinos, a quienes se responsabiliza de que exista un mercado con precios estancados desde hace casi una década. Buscan crecer tanto en el mercado europeo como en el indio, pero siempre preocupados con los competidores chinos.

También a fines de septiembre, igualmente a la espera de la aprobación de la UE, se alcanzó un memorándum de entendimiento entre la alemana Siemens y la francesa Alstrom, con participación minoritaria estatal, para formar una empresa competitiva en el sector ferroviario. Alstrom fue suficientemente explícita al explicar que, detrás de su decisión, se encuentra un "jugador dominante en Asia [que] ha cambiado las dinámicas del mercado global”, y es de igual importancia que la digitalización. ${ }^{20}$ Incluso con esta fusión la Chinese Railroad Company, mejor conocida por sus siglas, CRRC, seguirá siendo la principal empresa proveedora de equipo en el ramo, seguida por las ya mencionadas, con un volumen de ventas cercano a los 18 mil millones de euros. Debido a eso, se especula sobre la posibilidad de integrar posteriormente a la canadiense Bombardier y superar con ello tanto a la CRRC como a la japonesa Hitachi.

Como parte de movimientos complejos sobre quién posee cuáles líneas áreas, desdibujándose el viejo perfil de asociar líneas con países, China Eastern, con 751 millones de euros hablando por ella, y de manera similar a Delta, adquirió parte del paquete accionario de Air France-KLM.

En 2017, uno de los movimientos financieros relevantes ocurrió en la industria química. En el plano interno, Sinochem y ChemChina se fusionarían en 2018 y tendrían ingresos de

\footnotetext{
${ }^{20}<\mathrm{http}$ //www.alstom.com/press-centre/2017/09/siemens-and-alstom-join-forces-to-create-a-europeanchampion-in-mobility/>.
} 
100 mil millones de dólares. En el plano internacional, lo cual ya es un hecho, la primera de las dos empresas chinas mencionadas compró, por 43 mil millones de dólares, el 82\% de la suiza Syngenta, lo cual coloca a los chinos en una posición excelente en la producción de alimentos modificados, pesticidas, y en investigación en tecnología de alimentos.

En el nivel específicamente de las relaciones intergubernamentales, chinos y europeos, sobre todo en el gobierno de Angela Merkel, trabajan en diferentes vías. En una buscan formas de cooperar para enfrentar a Trump, lo cual, considerando la evolución en estilos y tácticas de negociación, parece haberles resultado más fácil a los chinos. En otra vía compiten, los alemanes incluso se oponen a Beijing, siempre sin dejar de cooperar.

Alrededor de una reunión con Xi, sostenida en junio de 2017, Merkel buscó llegar a un tratado de libre comercio, el cual podría estar precedido de un acuerdo en materia de inversión. Si bien han llegado a acuerdos de cooperación en Asia Central y a uno para la construcción conjunta de una hidroeléctrica en Angola, la alemana ha insistido en la necesidad de abordar el tema de los derechos humanos como una manera de profundizar las relaciones.

Macron, presidente de Francia, percibido como el más liberal de sus pares europeos, aparece como el orquestador de un bloque que busca que la UE adopte medidas para contener a China en la compra de empresas de sectores estratégicos como las que se han descrito. La presión del presidente francés y el apoyo recibido no se han materializado ahora en políticas específicas que impacten en lo que los chinos han venido haciendo dentro de las economías de la Unión Europea. De ninguna manera ha enfrentado a los chinos. Por el contrario, prepara un viaje a Beijing y le interesa continuar alentando las exportaciones aeroespaciales, hasta ahora las más importantes, pero también las de los bienes de consumo que esperan crezcan más.

El Reino Unido tiene varios impedimentos para acercarse institucionalmente a China. Uno de ellos es de orden político, la reacción que provocaría en la Casa Blanca: ¿qué tanto podría acercarse a China sin despertar la suspicacia de Trump? Esto podría cambiar después de los resultados favorables del estadounidense de su visita de noviembre de 2017, a Beijing. 
La otra razón es de orden institucional: mientras permanezca dentro de la Unión Europea, no podrá firmar acuerdos bilaterales, lo cual podría ser hasta $2019 .^{21}$

Boris Johnson, secretario de Asuntos Exteriores del RU, ha privilegiado los acercamientos con Beijing; incluso, como se describió antes, ha apoyado la iniciativa "un cinturón y un camino". Hay un gran interés de estar cerca de los chinos; podrían ser de gran ayuda en el futuro. Como otros gobiernos, éste, representado por Johnson, en consonancia con la Casa Blanca, se suma a las presiones para que Beijing ejerza mayor influencia sobre Corea del Norte.

Si bien Beijing explora las diferentes posibilidades que Londres le podría ofrecer, también actúa con cautela, sobre todo en el tema financiero. En 2016, los chinos escogieron a la capital del Reino Unido como lugar para ofrecer el equivalente a 430 de millones de dólares en bonos de renminbi. Sin embargo, ante la situación reinante, los responsables chinos dijeron desde inicios de año que consideraban que eso posiblemente no se volvería a repetir.

\section{CHINA Y RUSIA, LA AMISTAD IMPREDECIBLE}

Xi y Putin se encuentran de manera regular; tienen muchos espacios compartidos, además de los bilaterales. Uno de ellos es el de la Organización de Cooperación de Shanghai (OCS), donde coincidieron en junio de 2017. El significado de estos encuentros es que en ellos muestran que un sinnúmero de temas de la agenda global y bilateral tienen grandes coincidencias. La agenda de cada uno de ellos es precisa y particular, pero hay temas en los que trabajan en tándem. Incluso han hablado, sobre todo los rusos, de crear una moneda común y de integrar su sistema de pagos, el kartamir, al de los chinos. China se ha dedicado a cultivar la amistad con Rusia, enorme espacio esencial para que su principal política global tenga éxito.

Además de que se distancian de Trump respecto a Corea del Norte, fortalecen su cercanía por el intercambio de bienes diferentes, no siempre tangibles. Beijing colabora con

$21<$ http://uk.businessinsider.com/eu-warns-theresa-may-about-post-brexit-trade-deal-with-trump-usa2017-1>. 
los proyectos de Putin. En julio de 2017 le otorgó un fondo de inversión de 10 mil millones de dólares, con lo cual se fortalecerá la Unión Económica Euroasiática mediante la construcción de infraestructura lo que redundará en beneficio de la política insignia de Beijing, "un cinturón...”. Otro de los acuerdos firmados fue el relativo a destinar 882.35 millones de dólares para el desarrollo de investigación computacional de cadena de bloques.

Putin pone el teatro de operaciones y su legitimidad, Xi el dinero y el comercio. Ambas partes intercambiaron los mejores bienes a su disposición; China ofreció lo tangible y Rusia aparece como receptora de lo que Beijing le otorga financieramente, de lo cual carece Rusia. La relación se fortaleció en 2017 debido a la confluencia de necesidades complementarias, pero por las características de la alianza y la intención de Putin de contar con espacio ruso perfectamente definido, la impredictibilidad tomó forma definida en estos últimos meses. Parecería que, ante la mejora de la relación china con Trump a partir de noviembre de 2017, Beijing se alejaría de Moscú. En todo caso, su situación de negociación mejora y podrá obtener más de ambos.

\section{China en América Central y del Sur, AVANCES Y PROFUNDIZACIÓN DE RELACIONES}

\section{Panamá, un triunfo chino en la guerra de posiciones}

Año y medio después del triunfo de Tsai Ing-wen en las elecciones presidenciales taiwanesas y debido a su posición contraria al llamado Consenso de 1992, dentro del contexto de una guerra de posiciones en América Central, Beijing alentó a que Panamá se pronunciara a favor del llamado principio de una sola china y establecieran relaciones bilaterales diplomáticas, después de por lo menos una década de intentarlo sin éxito. Previo establecimiento de relaciones diplomáticas en junio y elevación de la oficina comercial a embajada, a mediados de septiembre de 2017 finalmente se abrió la sede diplomática china en el istmo.

Los lazos económicos son conocidos, sobre todo el hecho de que los chinos son los segundos usuarios en importancia del canal. Ahora la mira está puesta en convertir a Panamá en sitio turístico autorizado y recibir una parte del creciente número de turistas chinos. Un 
primer avance, en el contexto de la apertura de la sede diplomática, fue la firma de un memorándum de entendimiento para establecer rutas aéreas.

El cambio de estatus diplomático perjudicó a los sectores exportadores que se beneficiaban de la relación con Taiwán. Uno de los más afectados fue el de carne de bovino, pero se espera la recuperación y la exportación de otros productos al continente, como podrían ser los acuícolas. El establecimiento de relaciones, pese a algunos inconvenientes, es visto como positivo, sobre todo por el flujo de empresas chinas a Panamá en busca de diferentes productos. Una serie de recientes transacciones generaron ingresos a los panameños por 38 millones de dólares, dos menos que en $2016 .{ }^{22}$

\section{Macri y Beijing, viento en popa}

La relación durante 2017 fue incluso más fructífera de lo que fue en 2016, un año de resultados positivos para ambos. En mayo, Mauricio Macri viajó a China y regresó con muchos acuerdos firmados. Además de la declaración conjunta con su homólogo Xi y del acta de la segunda reunión de la Comisión Binacional Permanente, destacan la firma de un memorándum para la formación de diplomáticos, un acuerdo para el establecimiento de un centro cultural chino, un plan de acción estratégico en materia de cooperación agrícola, un plan de trabajo 2017-2019 para la cooperación en materia de inocuidad, sanidad y seguridad alimentaria, un protocolo de requisitos fitosanitarios para la exportación de uvas de mesa, un memorándum de entendimiento sobre cooperación en materia ferroviaria para el proyecto de recuperación del Ferrocarril San Martín de Cargas, una adenda al acuerdo entre el Ministerio de Transporte de la República Argentina y China Machinery Engineering Corporation (CMEC), un contrato del proyecto Jujuy Interconectada y Segura, una acta de ratificación de acuerdos para la construcción de la planta de energía renovable Cauchari Solar en la provincia de Jujuy, un memorándum de entendimiento sobre cooperación en materia de futbol, un acuerdo de cooperación entre la agencia de inversión y promoción del comercio internacional de Argentina y el Consejo para la Promoción del Comercio Internacional de China, un memorándum de entendimiento entre la oficina de promoción de la inversión y el comercio argentina y su par, la Oficina del Desarrollo del Ministerio de Comercio, un

\footnotetext{
${ }^{22}<\mathrm{https}$ ://www.forbes.com.mx/mision-comercial-de-china-en-panama-logra-transacciones-por-38-mdd/>.
} 
acuerdo financiero, otro en materia de impulso a la energía nucleoeléctrica, un plan quinquenal integrado para la cooperación en infraestructura 2017-2021, un acuerdo por canje de notas verbales para modificar el acuerdo sobre facilitación de visas de turismo, uno más por canje de notas verbales para modificar el acuerdo sobre facilitación de visas para viajeros de negocios, y un memorándum de entendimiento entre el Ministerio de Finanzas argentino y el China Development Bank. ${ }^{23}$

Desde la perspectiva de las relaciones bilaterales gubernamentales, la relación latinoamericana más fructífera fue con Argentina, al menos por la cantidad, variedad e importancia de los acuerdos, donde destaca una de las grandes preocupaciones gubernamentales chinas: su debilidad en el futbol, que esperan que los argentinos les ayuden a remontar.

Macri se ha esforzado por profundizar la relación con China y obtener de ella lo más posible, incluso más que su antecesora, Cristina Fernández de Kirchner, la cual él mismo acusó de ser demasiado cercana a Beijing. Su viaje es una muestra de lo exitoso que ha sido. No obstante, existen problemas que se originan en las formas y contenidos de la negociación. El más preocupante para algunos es el de una base en Neuquén en la Patagonia, sobre la cual hablamos en el Anuario 2016. La instalación despierta suspicacias en Argentina, pese a que tanto la Casa Rosada como Zhongnanhai aseguran que su uso será pacífico y científico. La razón de ello es que la administra el China Satellite Launch and Tracking Control General (CLTC), organismo bajo la dirección del Departamento General de Armamentos del Ejército Popular de Liberación. La queja principal es la presunta falta de transparencia de una instalación que desde febrero de 2017 estuvo lista para funcionar.

El problema que enfrentan los argentinos, como muchos otros, es el de la composición de los intercambios comerciales, donde los de mayor valor agregado son de procedencia china. Entre enero y agosto, lo cual complica el panorama comercial para los sudamericanos, sus ventas de frijol de soya bajaron $14.44 \%$ respecto al mismo periodo de 2016 , mientras que las de Uruguay se incrementaron $104.49 \%{ }^{24}$ Los sectores rentistas de varios países, entre

\footnotetext{
${ }^{23}<$ http://www.telam.com.ar/notas/201705/189339-acuerdos-argentina-china.html $>$.

${ }^{24}<\mathrm{http}$ ://www.lanacion.com.ar/2067523-por-menores-ventas-argentinas-la-soja-uruguaya-gana-lugaren-china $>$.
} 
ellos Brasil, compiten entre sí beneficiando a los consumidores chinos. Los uruguayos han estado trabajando muy de cerca con investigadores chinos para desarrollar soya diseñada específicamente para el consumo humano. En mayo de 2017, el proyecto entró en una nueva fase, ya que los uruguayos recibieron material genético conocido como germoplasma.

\section{China y Chile, hasta el infinito...}

Además de que ambos países están explorando esferas no tradicionales de la relación, como astronomía, fibra óptica y cooperación en la Antártica, a partir de mayo de 2017, durante una visita a China, ambas representaciones emprendieron la tarea de profundizar el tratado de libre comercio vigente con la forma de un memorándum de entendimiento. Algunos de los temas a discutir son los de las compras gubernamentales, servicios, comercio electrónico, problemas ambientales y competencia, entre otros. Además, el gobierno chileno ingresó como miembro del Banco Asiático de Inversión en Infraestructuras (BAII).

Asimismo, firmó con su contraparte china diez documentos de diferente naturaleza. Destacan, primero, al igual que con Argentina, las Actas de la I Reunión de la Comisión Binacional Permanente Chile-China. A continuación encontramos el acuerdo de cooperación en temas antárticos, un memorándum de cooperación estratégica, la renovación del Plan Quinquenal de Trabajo 2013-2017 en materia de cooperación agrícola, un convenio de requisitos sobre el transporte de mercancías de frutas de Chile al mercado de China a través de terceros países vía marítima y aérea, el Protocolo sobre ingreso de paltas al mercado chino, un memorándum de entendimiento entre el Ministerio de Economía y la parte china representada por la National Tourist Administration, un memorándum de cooperación e intercambio de información sobre mercados de capitales, un acuerdo de colaboración entre InvestChile y el Consejo Chino para la Promoción del Comercio Internacional (CCPIT), un memorándum de entendimiento para la creación de un centro conjunto de investigación en tecnologías de información entre la Universidad de Concepción y el Instituto Tecnológico de Harbin y DATANG Telecom. ${ }^{25}$

\footnotetext{
${ }^{25}<$ http://www.emol.com/noticias/Nacional/2017/05/13/858223/Los-diez-acuerdos-suscritos-entre-Chile- yChina-para-mejorar-el-comercio-y-el-apoyo-bilateral-en-turismo-ciencia-e-inversiones.html>.
} 
En un encuentro paralelo al de los líderes del APEC, el 11 de noviembre de 2017 en Dang Ang, Vietnam, chinos y chilenos firmaron un acuerdo para ampliar el tratado de libre comercio vigente desde 2015, mediante el cual integran servicios y economía digital.

\section{Brasil, terreno fértil para la inversión china}

De manera similar a Delhi, Brasilia y Beijing cuentan con el marco de los BRICS que les otorga un espacio para fortalecer sus lazos, el cual Xi aprovechó para insistir en su deseo de participar más dentro del Mercosur y que les facilitó la firma de 14 documentos de diferentes características y muy similares a los signados con argentinos y chilenos. Estuvo presente el nuevo tema, ya abordado con Macri, de la cooperación en materia de futbol. Además, comercio electrónico, facilitación de expedición de visas y seguridad alimentaria. China está dispuesta a invertir en la industria cinematográfica brasileña y a apoyar financieramente proyectos de energía y transporte.

El Banco de Desarrollo brasileño recibió un crédito de 3 mil millones de dólares; a su vez, el Banco de Brasil tendrá acceso a uno de 300 millones de dólares. El principal acuerdo alcanzado con Michel Temer, desde la perspectiva de los objetivos diplomáticos chinos, fue el de promover a un nuevo nivel la asociación estratégica, tema alrededor del cual gravitan los objetivos políticos de Beijing.

Ya en mayo de 2017, habían anunciado una inversión de 20 mil millones de dólares para impulsar el Fondo Brasil-China de Cooperación para la Expansión de la Capacidad Productiva, provenientes en su mayor parte, 15 mil millones, del Fondo Chino para la Cooperación para la Inversión en América Latina y el resto de la Caixa Econômica Federal y del Banco Nacional de Desenvolvimento Econômico e Social (BNDES). ${ }^{26}$

Además de las grandes cantidades en ayuda y cooperación, su inversión directa les ha permitido a los chinos afianzarse como los principales compradores en Brasil durante 2017. Los sectores energía, inmobiliario, software y comercio son algunos de los favoritos de los chinos.

\footnotetext{
${ }^{26}<$ https://oglobo.globo.com/economia/brasil-china-lancam-fundo-de-us-20-bi-para-financiarinfraestrutura-21414958>.
} 
Los exportadores brasileños de carne bovina enfrentaron en 2017 problemas en el mercado chino. ${ }^{27}$ Dos son las razones de las dificultades. Una está relacionada con argumentos de tipo sanitario, la llamada carne débil, que llevó a 20 países a restringir la importación desde este país sudamericano, restricciones que finalmente se levantaron tras un arduo trabajo de negociación de los brasileños. La otra es el acuerdo que permite la exportación de ese producto de origen estadounidense, con lo cual la competencia se incrementa.

\section{HONG KONG, 20 AÑOS SON NADA}

El 1 de julio de 2017 se cumplieron 20 años del inició de Hong Kong como parte de la soberanía china. Bajo el concepto de un país, dos sistemas, se creó la Región Administrativa Especial de HK: en lo político, sobre todo defensa y política exterior, Beijing es la fuerza dominante, tiene el lugar que antes ocupó el Reino Unido; en lo económico, las islas seguirían funcionando como hasta ahora, esencialmente la principal economía de libre mercado del planeta, sin la presencia del Estado chino. Las cosas no han resultado de la manera como la mayoría, tanto de locales como de foráneos, esperaba. Las fuerzas estatales chinas, sobre todo las relacionadas con la economía, han resultado vitales para evitar que las crisis financiares estructurales globales desbarranquen el proyecto hongkongenés.

A fines de los años ochenta e inicios de los noventa, miles de hongkongneses emigraron por la incertidumbre que les provocaba la cada vez mayor cercanía de la salida británica y la llegada del Ejército Popular de Liberación. No es que los británicos hubieran sido magnánimos y democráticos, pero los movimientos sociales chinos de 1989 y la represión de junio de 1989 en Tiananmén aparecían como una advertencia de lo que podría ser su futuro. Los migrantes fueron a varios países de la región, atraídos por gobiernos como los de Singapur y Taiwán, a donde llegaron entre 1000 y 3000 personas anualmente a asentarse, muchas para dedicarse a la producción de alimentos o a los servicios estéticos. Australia fue un destino privilegiado, al cual llegaron para 1991 casi 30 mil inmigrantes. Canadá fue el

\footnotetext{
${ }^{27}<$ http://www.minrel.gob.cl/con-10-acuerdos-y-el-ingreso-de-chile-al-banco-asiatico-de-inversiones/ minrel/2017-05-13/155112.html $>$.
} 
favorito con 278000 en ese mismo año. A Gran Bretaña arribaron alrededor de 100000 personas. No les interesaba regresar, al menos eso pensaron.

En 1990, el PIB per cápita en Hong Kong era de 13845 dólares y la población rondaba los 6 millones de habitantes. En 2016 el PIB per cápita fue de 43681 dólares y la población rebasó los 7 millones de habitantes. El haberse ido solamente hizo que algunos de esos migrantes se volvieran cosmopolitas y pudieran hacer negocios en un par de lugares del mundo al mismo tiempo, uno de ellos Hong Kong, lo cual ha sido facilitado por la integración económica a China y la posibilidad de acceso a mercados disímiles, pero mutuamente atractivos. En este plano, todo resultó mejor para muchos actores participantes en la economía. De hecho, la tan temida intervención estatal continental ha sido provechosa, sobre todo cuando ha servido para proteger a la economía isleña y a su moneda frente a los ataques especulativos. Es una de las economías que menos ha sufrido los embates de las crisis, ya sea estructurales o coyunturales.

Para mediados de 2017, el PIB había crecido 4.3\% y para septiembre 7\%. Ello ha sido posible gracias a bienes raíces, el incremento del empleo, la bolsa de valores y al crecimiento del comercio. ${ }^{28}$ Hasta el mes de agosto de 2017, el valor de las exportaciones se había incrementado $8 \%$ y los bienes importados crecieron $8.6 \% .{ }^{29}$ Hong Kong es un centro de libre comercio entre el mundo y China.

El dilema para los actores sociales sigue siendo el de la transmisión y repartición del poder. Algunos en Hong Kong desean que se elija a los gobernantes de forma universal, directa y secreta, y que el poder de decisión quede en manos de los hongkongneses. Otros, además, desean la independencia. No son los mismos y a veces son muy diferentes.

Para Beijing y sus aliados locales, debe ser una selección en que el gobierno central tenga la última palabra y la persona elegida de una terna no sea necesariamente la más popular entre la población. Para Beijing, su postura se ubica dentro del marco constitucional vigente; es mejor designar a sus contrapartes y socializarlos en sus métodos de negociación y proceso de toma de decisiones: el Estado chino homogeneiza a sus componentes. Fuera y en contra

\footnotetext{
$28<$ http://www.scmp.com/news/hong-kong/economy/article/2094139/hong-kong-economy-smashesforecasts-first-quarter-2017-grow $>$.

${ }^{29}<$ http://hongkongbusiness.hk/economy/news/hong-kongs-total-goods-exports-value-rose-74>.
} 
de esa socialización, es casi imposible formar parte de él. Es esencialmente refractario a fuerzas políticas centrífugas, las cuales no le son desconocidas, pero no le resultan aceptables.

Es refractario, pero no impenetrable. Los opositores han tomado las calles innumerables veces, lo hicieron desde fines de septiembre y el mismo 1 de octubre de 2017, la fiesta nacional más importante en el calendario político del Partido Comunista. Incluso, dirigentes opositores fueron llevados por el voto ciudadano al Consejo Legislativo. Algunos, como Joshua Wong, Alex Chow y Nathan Law, fueron apresados en julio de 2017, acusados de asociación ilegal, o el caso de Cheng Chung Tai, multado por profanar las banderas de Hong Kong y China.

Un medio japonés, ${ }^{30}$ Japan Times, ha destacado que el movimiento opositor, dividido en términos generales en prodemocracia e independentista, no está desarticulado; por el contrario, los liderazgos se han fortalecido y los jóvenes tendrán la edad suficiente para presentarse en las elecciones de enero de 2018.

El 26 de marzo de 2017, el Comité Electoral de la Región Administrativa de Hong Kong, que cuenta con 1194 miembros, designó a Carrie Lam Cheng Yuet-ngor con 777 votos de los 1 194, John Tsang Chun-wah obtuvo 365, Woo Kwok-hing 21 y 4 fueron invalidados. De haber sido una elección directa, el ganador hubiera sido Tsang, pero Lam contaba con el apoyo de los votos cooptados por Beijing, desde donde pronto le llegó la aprobación a través de Li Keqiang, primer ministro, a quien legalmente no le correspondería hacerlo.

Respecto a Lam, Tsang era más popular, mejor conectado en el mundo de los negocios y con posiciones más adecuadas en los ejecutivos anteriores; sin embargo, fue derrotado. La fortaleza de la primera mujer en ocupar el puesto político más importante de Hong Kong emana de sus relaciones con Beijing, las cuales se explican por la consistencia y firmeza que mostró contra el movimiento social en 2014, ante cuyos dirigentes jóvenes defendió el orden legal existente, así como por las negociaciones opacas y que no tomaban en cuenta a la ciudadanía en las que participó para tener un Museo del Palacio en Hong Kong que

\footnotetext{
${ }^{30}<$ https://www.japantimes.co.jp/news/2017/09/17/asia-pacific/politics-diplomacy-asia-pacific/jailingactivists-new-faces-step-hong-kong-democracy/\#.WdFv3BD4JKo $>$.
} 
satisficiera a la burocracia en Beijing, ${ }^{31}$ a la cual le ahorró tener que rendir cuentas y posiblemente enfrentar descontento por el proyecto de 3.5 mil millones de dólares hongkongeses. Además, facilitó que el arquitecto Rocco Yim Sen-kee se involucrara en el proyecto de forma poco clara.

Tsang hizo poco, o nada, para congraciarse con Beijing. Por el contrario, fue percibido como alguien susceptible de recibir influencia de parte de los prodemocracia, lo cual lo convirtió en poco confiable a los ojos del gobierno central.

El gobierno de la RPCh tiene todo a su favor para mantener el statu quo. Cuenta con los requisitos institucionales, materiales, legales e ideacionales para imposibilitar un cambio en el arreglo negociado con los británicos en los años noventa. Además, Hong Kong y una porción considerable del continente, no solamente la correspondiente a la provincia de Guangdong, dan forma a una región económica específica y diferenciada de otras de China; la integración con los vecinos del Sudeste se ha institucionalizado. Entre marzo de 2013 y el mismo mes de 2017, ASEAN y los hongkongneses negociaron un acuerdo de libre comercio en diez rondas, cuya conclusión se dio a conocer en septiembre del mismo año. Beijing tiene claro los alcances y aspectos flexibles de "un país, un sistema". Los isleños tienen toda la flexibilidad para negociar acuerdos internacionales, con lo cual todos los involucrados se beneficiarán. Gracias al Acuerdo de Sociedad Económica Cercana entre China y Hong Kong (2003), cerca de 21819 productos pueden acceder al mercado chino con arancel 0 , con lo cual el comercio con el Sudeste se ha incrementado. Este acuerdo es muy completo, cubre comercio en bienes y servicios, inversión, además de cooperación técnica y económica.

\section{El futuro visto desde 2017}

Para las elites políticas chinas, el futuro es inconcebible sin un territorio unificado, lo cual se ha logrado en gran medida con la integración de un territorio que incluye Xinjiang y Tibet, desde los años cincuenta, y el regreso a soberanía china de Hong Kong y Macao en 1997. En los últimos meses, sobre todo a partir de 2016, Beijing se ha concentrado en hacer suyas y más grandes algunas islas e islotes del Mar del Sur de China. En este panorama, solamente

\footnotetext{
$31<$ http://www.scmp.com/news/hong-kong/politics/article/2057262/carrie-lam-cuts-short-christmasleave-head-beijing-again>.
} 
haría falta Taiwán, cuyo debilitamiento se procesa desde diferentes ángulos: pérdida de reconocimiento diplomático, como pasó en junio de 2017, cuando Panamá estableció relaciones con Beijing alejándose de Taipei, con lo cual se obligó a los isleños a acercarse a Beijing.

Al elemento territorial como base esencial de la construcción de la sinidad, está el tema del idioma. En la capital china, en abril de 2017, el Ministerio de Educación y la Comisión Estatal del Idioma esperan que, para 2020, 80\% de la población hable putonghua (普通话), enfocando sus aspiraciones lingüísticas porcentuales en las llamadas minorías nacionales y en las zonas rurales, donde apenas 40\% habla la lengua común. La sinidad necesita un espacio y una lengua, por ello unos meses después, en julio, se lanzó una campaña para alentar a la población de Hong Kong a comunicarse en huayu (华语): escuchar, hablar, leer y escribir (ting shuo du xie 听说读写). La campaña, que busca ajustarse a la población local, no se refiere a la lengua como putonghua, sino como huayu, una idea que les pudiera resultar culturalmente aceptable a los locales, sin peso ideológico como comunista, sinificada a los estándares del sur. Sin embargo, la tarea es complicada. Las dificultades de imponer una lengua quedaron expuestas desde el primer día, cuando en su propaganda los promotores confundieron leer-dú-读 por mostrar falta de respeto-dú 渎.

El problema esencial es que siguen siendo dos mundos diferentes en demasiados aspectos, los cuales no pueden ser homogeneizados con leyes, cárcel o multas. Ambos son chinos, pero con diferentes racionalidades y percepciones autoidentitarias diametralmente opuestas sobre el pasado, pero también sobre el presente, y aún más sobre el futuro.

La lengua encabeza una lista larga de diferencias y dificultades. 